\title{
UV-induced photochemistry of methyl coumalate (methyl 2-pyrone-5-carboxylate) isolated in low-temperature inert matrices
}

\author{
I.D. Reva ${ }^{\text {a,* }}$, M.J. Nowak ${ }^{\text {b }}$, L. Lapinski ${ }^{\mathrm{b}}$, R. Fausto ${ }^{\mathrm{a}}$ \\ ${ }^{a}$ Department of Chemistry, University of Coimbra, Rua Larga, 3004-535 Coimbra, Portugal \\ ${ }^{\mathrm{b}}$ Institute of Physics, Polish Academy of Sciences, Al. Lotnikow 32/46, 02-668 Warsaw, Poland
}

Received 6 July 2006; in final form 7 August 2006

Available online 17 August 2006

\begin{abstract}
Photochemical transformations of methyl coumalate have been studied by matrix-isolation technique. Two photoreactions were induced by UV $(\lambda>295 \mathrm{~nm})$ light: isomerisation to the Dewar form and $\alpha$-bond cleavage leading to the open-ring aldehyde-ketene. The first photoprocess was found to be strongly dominating. Experimental evidence of photoreversibility of both photoisomerisation processes has been provided. Upon $\lambda>200 \mathrm{~nm}$ irradiation, the photochemically formed monomeric Dewar species underwent decarboxylation, with production of methoxycarbonyl-substituted cyclobutadiene. This antiaromatic photoproduct was experimentally observed for the first time. All the photoproduced species were identified by comparison of their IR spectra with the spectra calculated at the DFT(B3LYP)/6-311++G(d,p) level.
\end{abstract}

(C) 2006 Elsevier B.V. All rights reserved.

\section{Introduction}

The unique structural features of pyran-2-one ( $\alpha$-pyrone) and its derivatives encouraged organic chemists to extensive investigation of this class of compounds. The molecules of $\alpha$-pyrones are endowed with electrophilic and nucleophilic centres that are responsible for substitution, addition, ring transformation and photochemical reactions [1]. The $\alpha$-pyrone sub-unit is found in a number of natural products [2] showing a broad-spectrum biological activity. Such compounds are characterised by their ability of binding to specific protein domains and exerting biological effects [3]. A wide range of substituted $\alpha$-pyrones show remarkable inhibitory activity against bacteria, yeasts and fungi [4]. $\alpha$-Pyrone is an important sub-unit of psoralen. Psoralen has a potential to increase skin pigmentation $[5,6]$, moreover, in combination with UV irradiation it has been used over decades in the treatment of psoriasis and

\footnotetext{
${ }^{*}$ Corresponding author. Fax: +351239827 703.

E-mail address: reva@qui.uc.pt (I.D. Reva).
}

other dermatoses [7-11]. Thus, the knowledge of the UV-induced photochemistry of $\alpha$-pyrone and substituted $\alpha$-pyrones may shed some light on the mechanisms of their biological activity.

Photochemical transformations of $\alpha$-pyrones may occur in two directions. The ring-opening photochannel involves the $\alpha$-cleavage of the $\mathrm{C}-\mathrm{O}$ bond and the formation of conjugated aldehyde ketene. An alternative photochannel results in valence isomerisation, with production of a Dewar structure (Scheme 1). The Dewar isomer, in turn, can undergo decarboxylation with a formation of antiaromatic cyclobutadiene.

In the 70s, photochemistry of the parent compound ( $\alpha$-pyrone) isolated in low-temperature matrices of inert gases was extensively studied. The ring-opening photochannel strongly dominates over the Dewar formation in a non-substituted $\alpha$-pyrone [12-17]. Addition of substituents may alter the photochemistry of the pyrone ring. For instance, the presence of methyl groups at positions 4 and 6 favours the valence isomerisation photochannel and formation of the Dewar analogue $[18,19]$. The same 


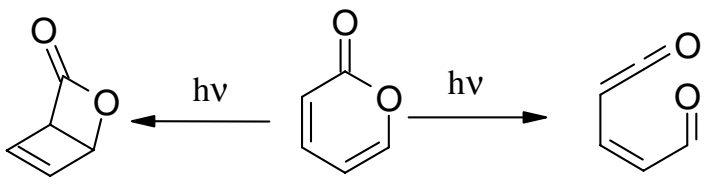

Scheme 1. Photochemical transformations of $\alpha$-pyrones.

trend has been found for 4,6-diphenyl- $\alpha$-pyrone [20] as well as for substituted 6-methyl-, 4-hydroxy- and 4-methoxy- $\alpha$ pyrones [21]. The synthesis and reactivity of different substituted pyrones have been comprehensively reviewed by Kvita and Fischer [22,23].

Among the different substituted analogues of $\alpha$-pyrone, the 5-substituted species have been studied to a lesser extent. To the best of our knowledge, only one report [24] deals with the solution and solid phase photochemistry of $\alpha$-pyrone bearing the carboxylate or ester substituent in position 5. Photochemistry of these $\alpha$-pyrone derivatives has been studied for the compounds dissolved in hydroxylic and nonhydroxylic solvents as well as for the solutions in heavy-atom solvents. Further investigations concerned photochemical transformations of $\alpha$-pyrone in a solid phase: suspended in $\mathrm{KBr}$ pellets or sandwiched between two quartz plates. Those experimental results turned out to be dependent both on the physical state and the solvent, as well as on the wavelength of the irradiating light. In order to explain those phototransformations, the authors frequently assumed an aggregation of the studied molecules. However, the importance of unimolecular photochemistry remained unclear.

The present study of the 5 -substituted $\alpha$-pyrone, methyl 2-pyrone-5-carboxylate (methyl coumalate), continues the ongoing investigation of UV-induced photochemistry of this class of molecules isolated in low-temperature inert matrices. Another purpose of this study is to distinguish between the photochemistry of 5 -substituted $\alpha$-pyrone monomers as opposed to their condensed state photochemistry [24], where interaction with solvents or self-aggregation was non-negligible.

\section{Experimental and computational methods}

The sample of methyl 2-pyrone-5-carboxylate used in the present study was a commercial product supplied by Aldrich. Even at a room temperature the saturated vapour pressure of the solid compound is sufficient for deposition of the matrices. The vapours of the compound (typically at $20^{\circ} \mathrm{C}$ ) were deposited, together with a large excess of an inert gas (argon or xenon), on a CsI window cooled to $10 \mathrm{~K}$. The experiments were carried out on two matrix-isolation setups described elsewhere $[25,26]$. The results obtained using each of the systems were the same.

The infrared spectra were recorded with $0.5 \mathrm{~cm}^{-1}$ resolution using either a Mattson (60AR Infinity Series) [25] or a Thermo Nicolet Nexus 670 FTIR [26] spectrometer, equipped with $\mathrm{KBr}$ beam splitters and DTGS detectors.
The matrices were irradiated with the light from an $\mathrm{HBO}$ 200 high-pressure mercury lamp fitted with a water filter and the Schott WG295 or Schott UG11 cut-off filters transmitting the light with $(\lambda>295 \mathrm{~nm})$ or $(\lambda>270 \mathrm{~nm})$, respectively.

The geometries of the isomers of methyl coumalate (as well as the geometries of the photoproducts derived from this compound) were optimised using the hybrid HartreeFock and density functional theory method DFT(B3LYP) with the Becke's three-parameter exchange functional [27], gradient-corrected functional of Lee, Yang, and Parr [28], and Vosko, Wilk, and Nusair correlation functional [DFT(B3LYP)] [29]. At the optimised geometries, the DFT(B3LYP) harmonic vibrational frequencies and IR intensities were calculated. All quantum-mechanical calculations were performed with the GAUSSIAN 98 program [30] using the standard $6-311++\mathrm{G}(\mathrm{d}, \mathrm{p})$ basis set. To correct for the systematic shortcomings of the applied methodology (mainly for anharmonicity), the predicted vibrational wavenumbers were uniformly scaled by 0.978 .

\section{Results and discussion}

The geometry optimisations, carried out in the present work for methyl coumalate (mp5c), led to identification of two minima on the potential energy surface. These minima correspond to structures $\mathbf{m p 5 c I}$ and $\mathbf{m p 5 c I I}$ presented<smiles>COC(=O)c1ccc(=O)oc1</smiles>
$\mathrm{mp} 5 \mathrm{cI}$<smiles>COC(=O)C1=CC2C(=O)OC12</smiles>
Dmp5cI<smiles>COC(=O)C1=CC=C1</smiles>
$\operatorname{cbI}$<smiles>COC(=O)/C(C=O)=C/C=C=O</smiles><smiles>COC(=O)c1ccc(=O)oc1</smiles>
$\mathrm{mp} 5 \mathrm{cII}$<smiles>COC(=O)C1=CC2C(=O)OC12</smiles>

Dmp5cII<smiles>COC(=O)C1=CC=C1</smiles>

cbII

aldehyde-ketene, several conformers
Scheme 2. Structures and abbreviated names of molecules considered in this work. 
in Scheme 2. Both mp5cI and mp5cII forms have planar pyran rings and are of overall $\mathrm{C}_{\mathrm{s}}$ symmetry. The electronic energy (including the zero-point vibrational contribution) of the mp5cI isomer was calculated [at the DFT(B3LYP)/ $6-311++\mathrm{G}(\mathrm{d}, \mathrm{p})$ level] to be lower by $1.54 \mathrm{~kJ} \mathrm{~mol}^{-1}$ than that of the mp5cII conformer. Because of the small energy difference, both forms should be expected to be populated in the gas phase. At $20^{\circ} \mathrm{C}$, the predicted populations of forms mp5cI and mp5cII are $65 \%$ and $35 \%$, respectively. The energy barrier separating these forms was predicted to be higher than $37 \mathrm{~kJ} \mathrm{~mol}^{-1}$ in both directions (estimated at the DFT(B3LYP)/6-31G(d,p) level). Such a high barrier precludes interconversions between the two conformers during their landing and cooling on the surface of the low-temperature matrix. This implies that the relative populations of the mp5cI and mp5cII isomers of methyl coumalate trapped into Ar and Xe matrices should be the same as they were in the gas phase prior to deposition [25].

The experimental IR spectra of monomers of mp5c isolated in argon and xenon matrices are presented in Fig. 1 (traces $\mathrm{d}$ and c). These spectra are well reproduced by the spectrum simulated on the basis of theoretical calculations carried out [at the DFT(B3LYP)/6-311++G(d,p) level] for isomers mp5cI and mp5cII (trace B). As it can be seen in Fig. 1 (trace a), the theoretical spectra of the two isomers of mp5c are very similar. Hence, it would be very difficult to distinguish between these forms in the experimental IR spectrum. Fortunately, the exact knowledge concerning the conformational orientation of the methyl-ester $\left(-\mathrm{COOCH}_{3}\right)$ group does not seem to be essential for the studies of photochemical transformations of mp5c.

Upon UV $(\lambda>295 \mathrm{~nm})$ irradiation of matrix-isolated mp5c the intensities of the bands of this compound decreased significantly (Fig. 2, traces a and b). In the emerging spectrum of the photoproduct(s), the appealing feature is the band at $1852 \mathrm{~cm}^{-1}$ (Ar), $1844 \mathrm{~cm}^{-1}$ (Xe). Such a wavenumber is typical of the bands due to the stretching vibrations of $\mathrm{C}=\mathrm{O}$ groups directly attached to small rings (e.g. the four-membered rings of the Dewar isomers). The frequency of the stretching vibration of the $\mathrm{C}=\mathrm{O}$ group attached to the six-membered pyran ring of mp5c is much lower: $1781 \mathrm{~cm}^{-1}(\mathrm{Ar}), 1775 \mathrm{~cm}^{-1}$ (Xe). Analogous pairs of bands due to $\mathrm{C}=\mathrm{O}$ stretching vibrations in the Dewar and Kekulé type structures were previously observed for other $\alpha$-pyrones: 1846 and $1757 \mathrm{~cm}^{-1}$ (Xe) for $\alpha$-pyrone itself [17], 1838 and $1761 \mathrm{~cm}^{-1}$ (Ar) for 4,6-dimethyl- $\alpha$-pyrone [19], 1828 and $1757 \mathrm{~cm}^{-1}$ (Ar) for 4-methoxy-6-methyl- $\alpha$-pyrone [21], 1838 and $1766 \mathrm{~cm}^{-1}$ (Ar) for 4-hydroxy-6-methyl- $\alpha$-pyrone [21]. This fact strongly suggests that also in the case of mp5c the Dewar

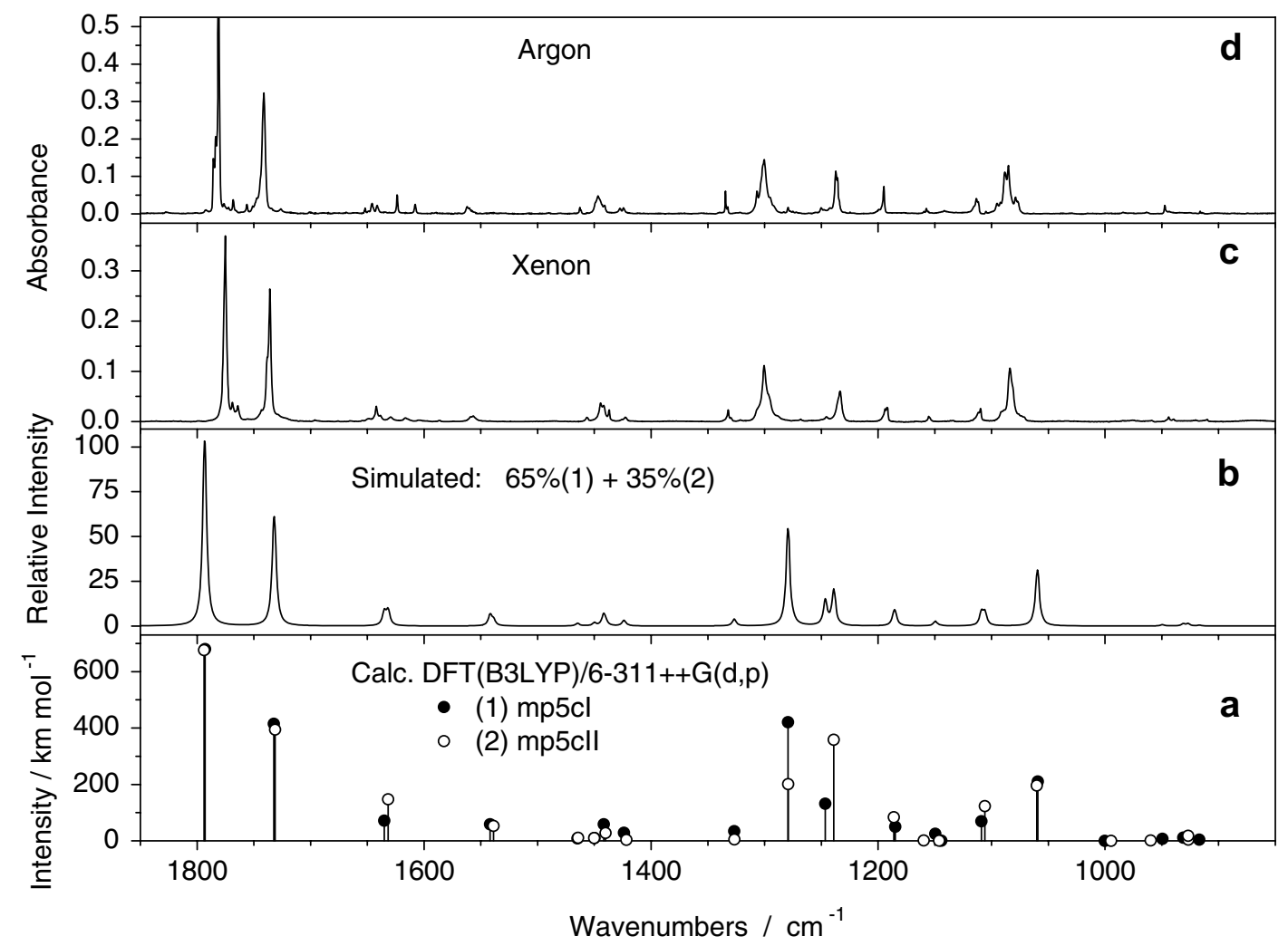

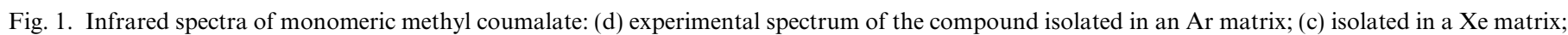

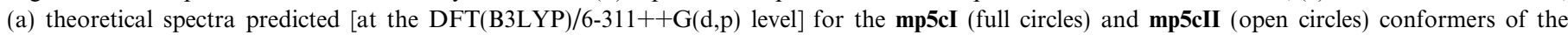

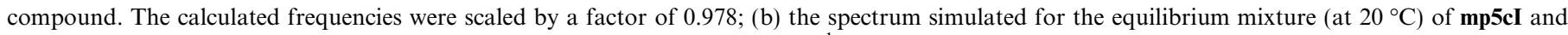

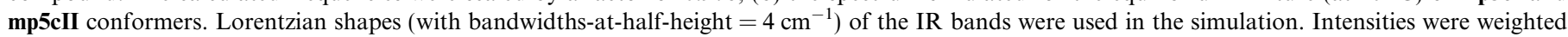
on the basis of the relative populations of $\mathbf{m p 5} \mathbf{c I}$ and $\mathbf{m p 5} \mathbf{c I I}$ conformers, corresponding to the calculated energy difference $\left(1.54 \mathrm{~kJ}\right.$ mol $\left.{ }^{-1}\right)$. 


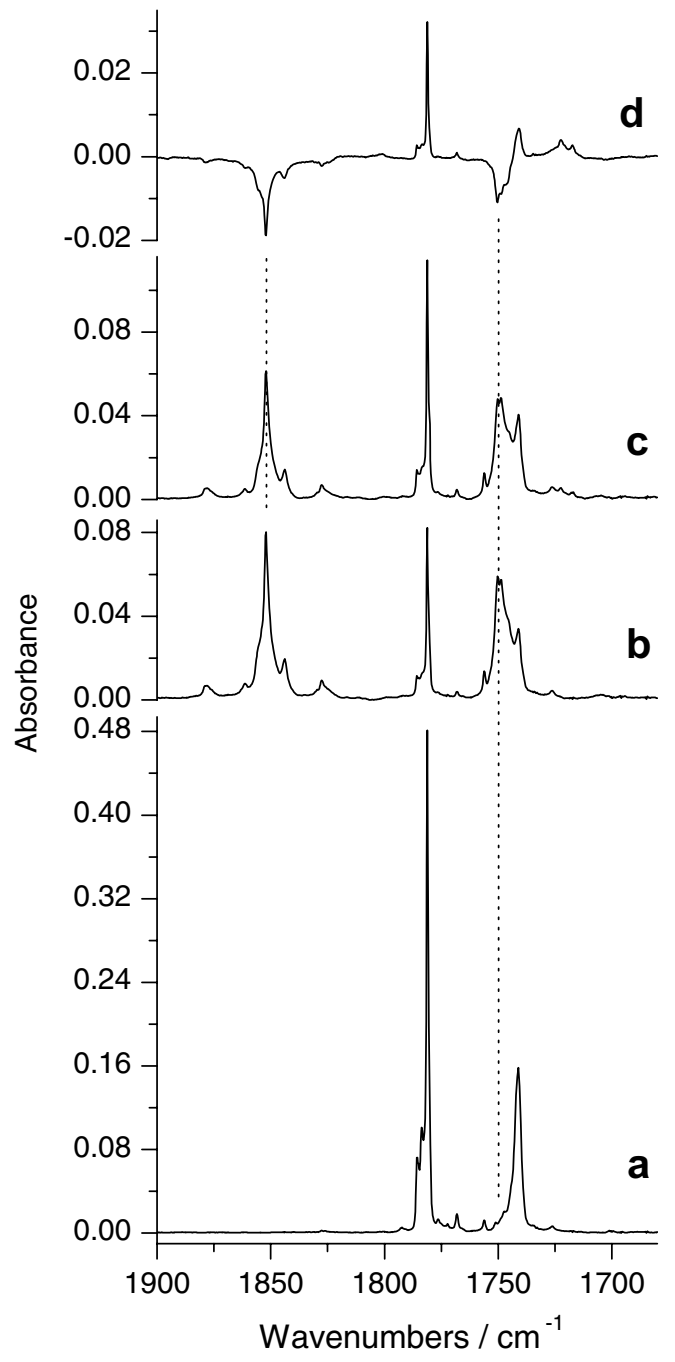

Fig. 2. UV-induced generation of the Dewar isomer (Dmp5c) of methyl coumalate and experimental evidence of photoreversibility of this process. Bands marked with dotted vertical lines are due to the Dmp5c photoproduct. (a) Infrared spectrum recorded after deposition of monomeric mp5c into an Ar matrix (10 K); (b) spectrum recorded after $100 \mathrm{~min}$ of irradiation with UV $(\lambda>295 \mathrm{~nm})$ light; (c) spectrum recorded after subsequent $25 \mathrm{~min}$ of UV $(\lambda>270 \mathrm{~nm})$ irradiation; (d) difference spectrum: trace (c) minus trace (b). Note that the ordinate scales (a):(b):(c):(d) relate as $0.5: 1: 1: 2$.

form of the compound would be generated upon an exposure of the matrix to $\mathrm{UV}(\lambda>295 \mathrm{~nm})$ radiation. Another band ascribable to the stretching vibration of a $\mathrm{C}=\mathrm{O}$ group was observed in the spectrum recorded before irradiation at $1741 \mathrm{~cm}^{-1}(\mathrm{Ar}), 1736 \mathrm{~cm}^{-1}(\mathrm{Xe})$. This band should be assigned to the stretching vibration of the $\mathrm{C}=\mathrm{O}$ group of the methylcarboxy $\left(-\mathrm{COOCH}_{3}\right)$ fragment. As expected, the frequency of this vibration did not change much with the transformation of the pyran ring into its Dewar analogue. In the spectrum recorded after UV $(\lambda>295 \mathrm{~nm})$ irradiation (see Figs. 2 and 3), the corresponding new band appeared at $1749 \mathrm{~cm}^{-1}(\mathrm{Ar}), 1741 \mathrm{~cm}^{-1}(\mathrm{Xe})$.

The main photoproduct generated upon the UV $(\lambda>295 \mathrm{~nm})$ irradiation of matrix-isolated $\mathbf{m p 5 c}$ was posi- tively identified as the Dewar form of the compound. This structure assignment was based on the comparison of the experimental spectrum of the photoproduct with that theoretically simulated for the Dewar isomer (Fig. 3). Just like it was for the Kekule type structure of $\mathbf{m p 5 c}$, two forms of the Dewar isomer (differing by the orientation of the $-\mathrm{COOCH}_{3}$ fragment with respect to the ring) had to be taken into consideration. These two structures, Dmp5cI and Dmp5cII, are presented in Scheme 2. The comparison of the experimental and theoretical spectra illustrated in Fig. 3 suggests that both Dmp5cI and Dmp5cII forms of the Dewar isomer were photogenerated.

The irradiation of matrix-isolated methyl coumalate with UV $(\lambda>295 \mathrm{~nm})$ light did not lead to a total consumption of the initial substrate and its transformation into the photoproduct(s). This could suggest that a photostationary state had been achieved. A photochemical reaction can lead to a photostationary state only if (alongside the reaction in question) there is a simultaneous photoreaction in the opposite direction. The occurrence of the photoreaction transforming the Dewar isomer back into the Kekulé structure of $\mathbf{m p 5 c}$ was experimentally evidenced by a sequence of irradiations of the matrix: first with UV $(\lambda>295 \mathrm{~nm})$ light (Fig. 2, trace b) and then with UV $(\lambda>270 \mathrm{~nm})$ light (Fig. 2, trace c). Upon the latter $(\lambda>270 \mathrm{~nm})$ irradiation, the bands due to the previously photogenerated Dewar isomer decreased, whereas those due to the initially deposited $\mathbf{m p 5 c}$ increased, demonstrating a partial repopulation of this species (see Fig. 2, traces $b, c$ and d). These intensity changes clearly evidence that not only the photoreaction transforming mp5c into its Dewar isomer, but also the photoreaction in the opposite direction occurs upon UV irradiation. These results also demonstrate that the position of the resulting photostationary state can be shifted by using UV light of different wavelengths for irradiation of the matrix.

The production of the Dewar isomer was not the only photoreaction observed. As it was mentioned in Section 1, the studied class of compounds ( $\alpha$-pyrones) can also undergo photochemical cleavage of the $\mathrm{C}-\mathrm{O} \alpha$-bond. Such photochemical reaction results in formation of the openring conjugated aldehyde-ketene (Scheme 2). Alongside the Dewar photoproduct, this species was formed also for mp5c studied in the present work (see Fig. 4). The clear spectral signature of the photogenerated aldehyde-ketene is the band appearing at a very characteristic frequency ca. $2130 \mathrm{~cm}^{-1}$. This band (shown in Fig. 4, where it is marked by ' $\mathrm{K}$ ') is due to the 'antisymmetric' stretching vibration of the $\mathrm{C}=\mathrm{C}=\mathrm{O}$ group. The aldehyde-ketene open-ring molecule, produced from $\mathbf{m p 5 c}$, has five conformational degrees of freedom, which can result in up to 32 conformers. A theoretical investigation of all possible structures of aldehyde-ketene conformers is too expensive to be carried out using a high-level method of quantum chemistry. In the current study, the structures of 12 open-ring conformers (with the $\mathrm{O}=\mathrm{C}-\mathrm{O}-\mathrm{CH}_{3}$ axis in cis orientation) were optimised at moderate theory level [DFT(B3LYP)/ 


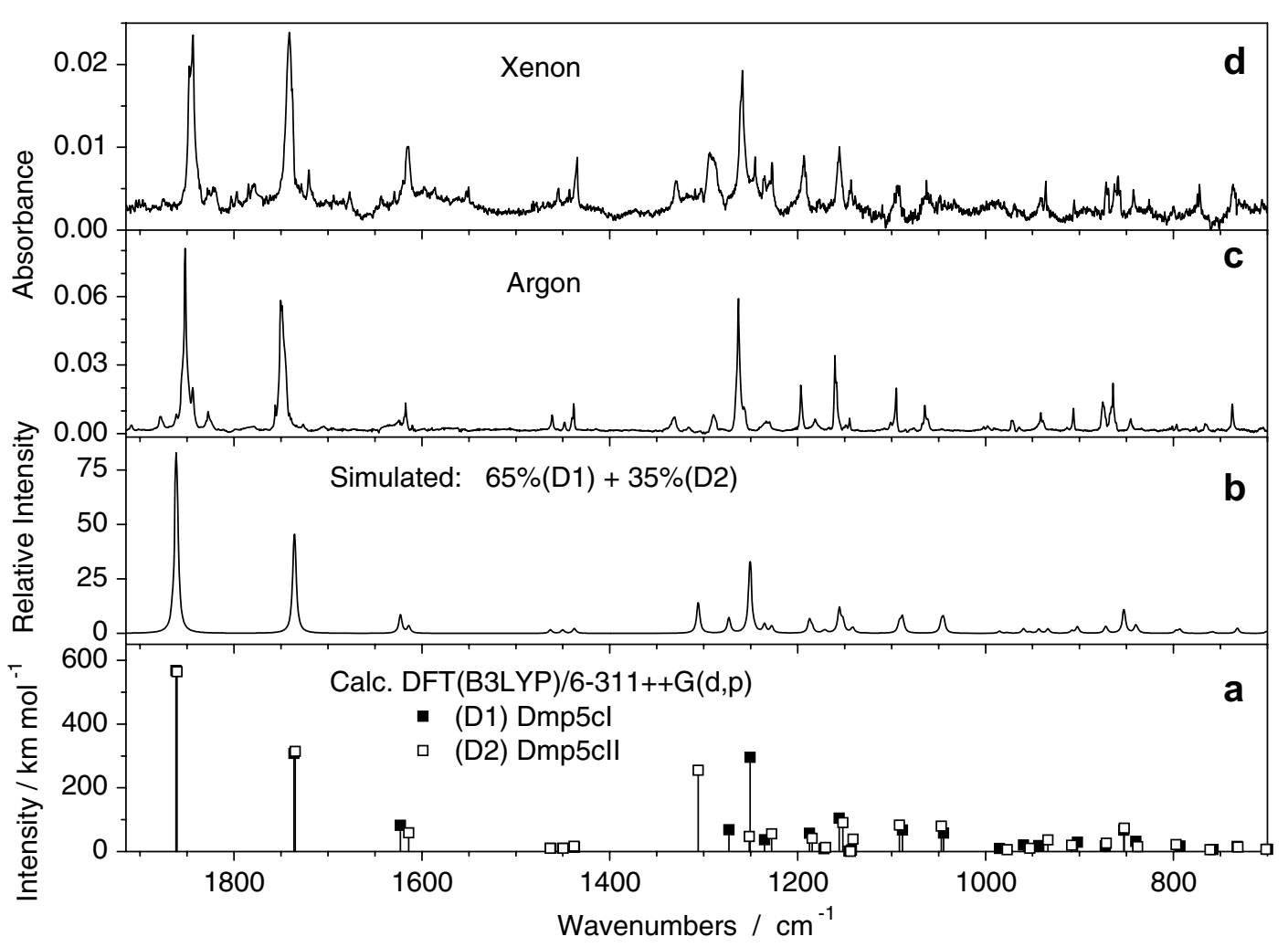

Fig. 3. Extracted experimental IR spectra of the Dewar form of mp5c isolated in argon (c) and xenon (d) matrices at $10 \mathrm{~K}$. The Dewar isomer was photogenerated upon UV $(\lambda>295 \mathrm{~nm})$ irradiation of the matrices. (a) Theoretical spectra predicted (at the DFT(B3LYP)/6-311++G(d,p) level) for the Dmp5cI (full squares) and Dmp5cII (open squares) conformers of the Dewar photoproduct. The calculated frequencies were scaled by a factor of 0.978; (b) the spectrum simulated, for the mixture of Dmp5cI and Dmp5cII conformers. Lorentzian shapes (with bandwidths-at-half-height $=4 \mathrm{~cm}^{-1}$ ) of the IR bands were used in the simulation. Intensities were arbitrarily weighted, with the same scaling factor as that used in frame (b) of Fig. 1.

6-31G(d,p)]. The geometry optimisations were then followed by calculations of the infrared spectra. For all these structures, the calculated frequencies of the antisymmetric $\mathrm{C}=\mathrm{C}=\mathrm{O}$ stretching vibration fall into a narrow range: $2192-2229 \mathrm{~cm}^{-1}$ (unscaled frequencies). It is then clear that it is practically impossible to experimentally distinguish between different conformers of the open-ring aldehydeketene on the basis of their spectral signatures.

In all the considered conformers of the aldehyde-ketene, the theoretically calculated intensity of the IR band due to 'antisymmetric' $\mathrm{C}=\mathrm{C}=\mathrm{O}$ stretching vibration is characteristically high. This value ranges from 950 to $1415 \mathrm{~km} \mathrm{~mol}^{-1}$, the average value being $1220 \mathrm{~km} \mathrm{~mol}^{-1}$. Using this prediction, it is possible to estimate the amount of the open-ring photoproduct in the matrix samples. From the viewpoint of the present study, especially interesting is the comparison of the efficiencies of the two alternative photochannels: ring-opening and valence isomerisation to the Dewar form. In order to estimate the amount of the Dewar photoproduct, the band due to the stretching vibration of the $\mathrm{C}=\mathrm{O}$ group attached to the four-membered ring (observed at $1852 \mathrm{~cm}^{-1}$ (Ar), $1844 \mathrm{~cm}^{-1}$ (Xe)) can be used, since it does not overlap with any band due to other species. The calculated intensities of the IR bands due to this vibration in the Dmp5cI and Dmp5cII isomers are equal to 564 and $568 \mathrm{~km}$ $\mathrm{mol}^{-1}\left(566 \mathrm{~km} \mathrm{~mol}^{-1}\right.$ in average). The experimental inte- grated intensities of the characteristic bands (due to the $\mathrm{C}=\mathrm{C}=\mathrm{O}$ 'antisymmetric' and $\mathrm{C}=\mathrm{O}$ stretching vibrations) in the IR spectra of the two photoproducts (aldehydeketene and Dmp5c, respectively) were measured at different stages of irradiation. Then these experimental values were divided by the calculated intensities of the corresponding theoretical bands. It was found that after a prolonged UV $(\lambda>295 \mathrm{~nm})$ irradiation (see trace a in Fig. 4), the amount of the Dewar isomer present in the matrix turned out to be seven times higher than the amount of the open-ring photoproduct. At all stages of irradiation, the share of the openring photoproduct was no higher than a few percent of the total. Noteworthy, the populations of the Dewar and aldehyde-ketene photoproducts were not growing parallel throughout the whole period of irradiation. The Dewar isomer was slowly accumulating in the sample, its amount increasing monotonously during several hours of irradiation. Instead, the amount of the open-ring aldehyde-ketene in the matrix increased very quickly within the first few minutes of the irradiation and afterwards it changed only very slightly. This behaviour suggests that a photostationary state between the starting form of the compound and the open-ring aldehyde-ketene product had been achieved upon just a few minutes of UV $(\lambda>295 \mathrm{~nm})$ irradiation. Then, together with the consumption of the initial substrate (by its transformation into the Dewar form), the population 


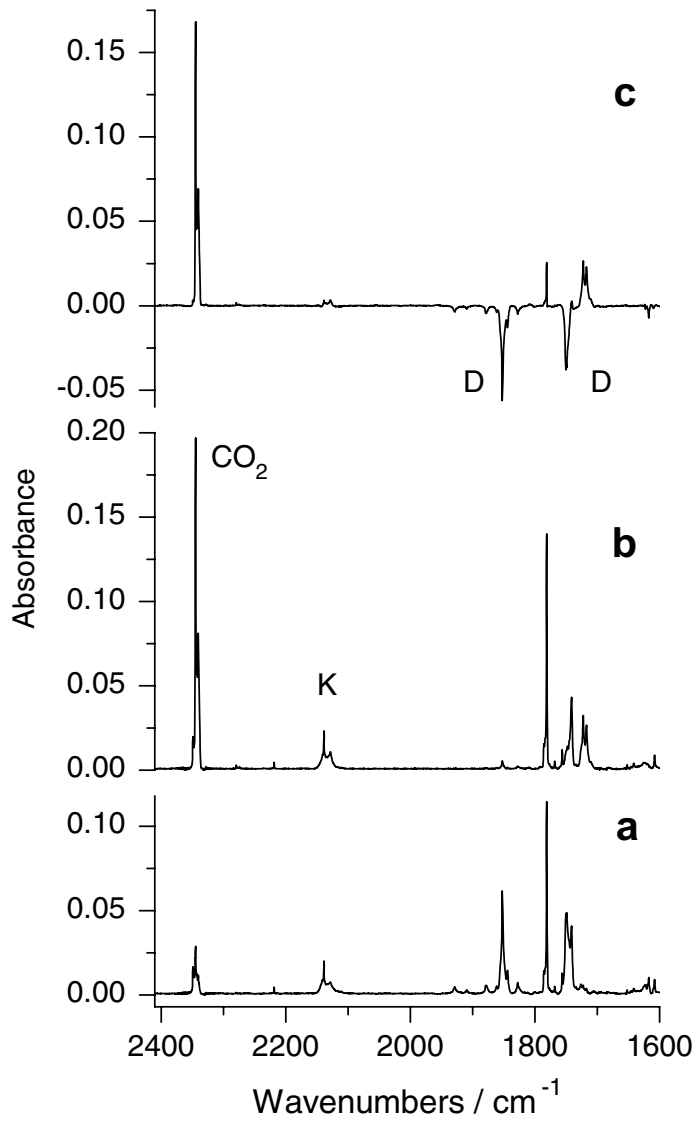

Fig. 4. (a) Experimental IR spectrum recorded after 100 min irradiation with UV $(\lambda>295 \mathrm{~nm})$ light plus $25 \mathrm{~min}$ of $\mathrm{UV}(\lambda>270 \mathrm{~nm})$ irradiation of monomeric mp5c isolated in an Ar matrix (this spectrum is the same as trace (c) of Fig. 2); (b) spectrum recorded after subsequent 5 min of UV $(\lambda>200 \mathrm{~nm})$ irradiation of the matrix; (c) difference spectrum, trace (b) minus trace (a). Abbreviations: ' $\mathrm{K}$ ' - open-ring aldehyde-ketene photoproduct; 'D' - Dewar photoproduct.

of the aldehyde-ketene (being in the dynamic 'ring openingring closure' photoequilibrium with $\mathbf{m p 5 c}$ ) was slowly decreasing. This also means that the ring-opening photoreaction is a photoreversible process.

The exposure of the matrices to the higher energy UV $(\lambda>200 \mathrm{~nm})$ light opened an additional photochannel. The appearance of a very strong new absorption band due to $\mathrm{CO}_{2}$ (see Fig. 4, trace b) demonstrates that the UV-induced decarboxylation had taken place under these conditions. Simultaneously with the growth of the characteristic IR band due to $\mathrm{CO}_{2}$, the IR absorptions due to the Dewar isomer Dmp5c decreased in intensity (see the difference spectrum $\mathrm{c}$ in Fig. 4; the bands pointing down and marked with ' $D$ ' are due to Dmp5c). This irreversible process led to nearly complete consumption of the Dewar isomer. Interestingly, upon the irradiation with the UV $(\lambda>200 \mathrm{~nm})$ light the bands due to the initial isomer of mp5c did not decrease. Instead, the intensities of these bands, such as that at $1781 \mathrm{~cm}^{-1}$ (Ar), clearly increased. This observation provides a further evidence of the reversibility of the photoreaction transforming mp5c into its
Dewar isomer. Moreover, this shows that the species undergoing decarboxylation induced by the UV $(\lambda>200 \mathrm{~nm})$ light is the Dewar isomer of methyl coumalate.

If $\mathrm{CO}_{2}$ is split from Dmp5c, then the expected product is methylcarboxy-cyclobutadiene (cb) (see Scheme 2). The generation of such an antiaromatic product was previously considered in the study on the photochemical and pyrolytical transformations of solid $\mathbf{m p 5 c}$ in $\mathrm{KBr}$ pellets [24]. Although only the evolution of $\mathrm{CO}_{2}$ was observed and no positive evidence of obtaining $\mathbf{c b}$ was provided, the authors (Javaheripour and Neckers [24]) claimed that the source of $\mathrm{CO}_{2}$ is not methyl coumalate itself but its dimers. The experimental work, carried out within the present study on matrix-isolated molecules of $\mathbf{m p 5 c}$, unequivocally demonstrates that the UV-induced decarboxylation concerns monomers of the Dmp5c reactant. The previous attempts to obtain methylcarboxy-cyclobutadiene (cb) [24] had failed, most probably because of the high reactivity of this antiaromatic species. One of the advantages of the matrixisolation technique is that such reactive species can be trapped in an inert, low-temperature environment and identified at leisure using stationary spectroscopic methods. That is why, in the present matrix-isolation study, it was possible to photogenerate, stabilise and spectroscopically identify the expected cyclobutadiene photoproduct.

The extracted experimental IR spectrum of the methylcarboxy-cyclobutadiene (cb) photoproduct is shown in Fig. 5. The molecule of cb can exist in two conformations: cbI and cbII (see Scheme 2). The theoretical spectra calculated for these two conformations are very similar to each other. Both of them are in a fair agreement with the experimental IR spectrum (see Fig. 5). However, it is very difficult to distinguish between these conformations or choose one of them, similarly as it was the case for the conformers mp5c and Dmp5c. Additionally, it can be noted that the two conformers, cbI and cbII, can interconvert between each other via the so-called automerisation mechanism, involving fast tunneling exchange between the $\mathrm{C}-\mathrm{C}$ and $\mathrm{C}=\mathrm{C}$ bonds in the four-membered carbon ring. The possibility of such process to occur in a low-temperature argon matrix has been demonstrated by Redington [31] for the non-substituted cyclobutadiene molecule. The possibility of a similar transformation can be suggested also for a substituted cyclobutadiene, such as methylcarboxy-cyclobutadiene.

The experimental observation of the cb photoproduct is also a clear indication of the unimolecular decarboxylation mechanism of the Dewar isomer and gives the evidence in favour of the fact that decarboxylation occurs from the Dewar substructure and not from the methylcarboxy substituent. Indeed, the position of the band due to the stretching vibration of the $\mathrm{C}=\mathrm{O}$ group observed in the spectra of the products of photodecarboxylation is similar to the frequencies of the stretching vibrations of the $\mathrm{C}=\mathrm{O}$ groups in the $\mathrm{COOCH}_{3}$ fragment of both mp5c and Dmp5c, but is considerably lower (by ca. $100 \mathrm{~cm}^{-1}$ ) than the frequency of the stretching vibration of the $\mathrm{C}=\mathrm{O}$ group directly attached to the four-membered ring of the Dewar isomer. 


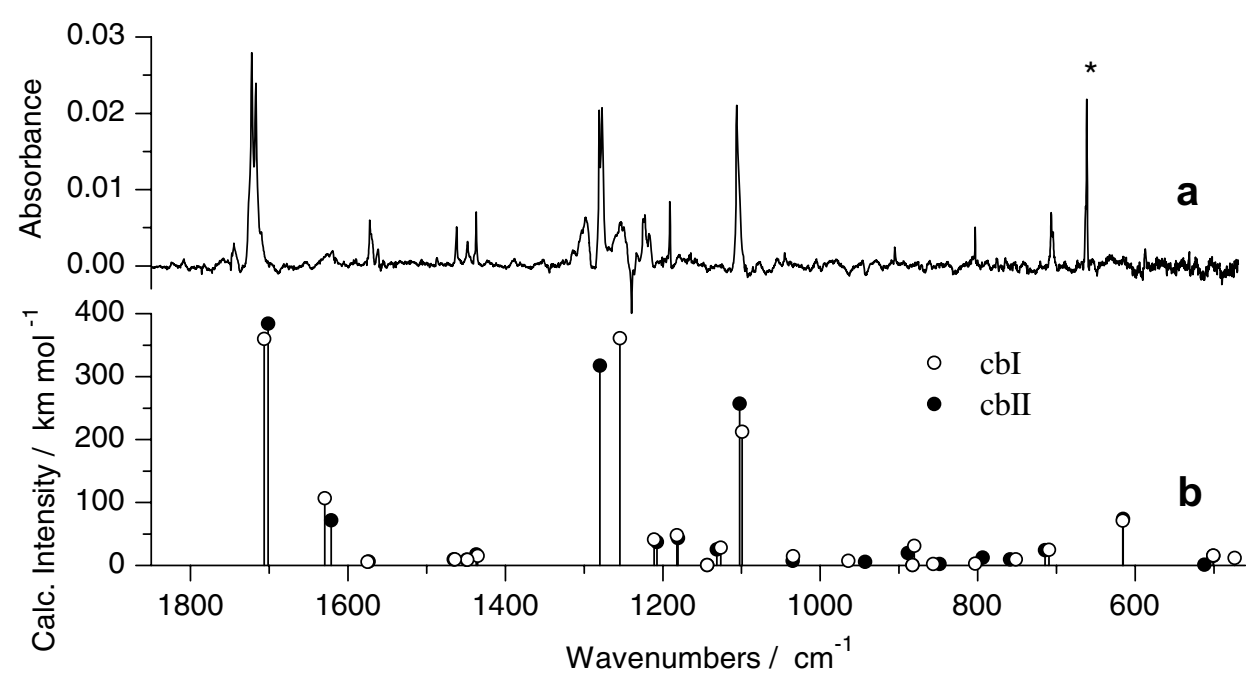

Fig. 5. (a) Extracted experimental IR spectrum of the product(s) of photodecarboxylation of the Dewar form of mp5c isolated in an Ar matrix at $10 \mathrm{~K}$. Photodecarboxylation was induced by irradiation of the matrix with UV $(\lambda>200 \mathrm{~nm})$ light. Asterisk designates the band due to $\mathrm{CO}_{2}$ molecule trapped in matrix together with the cb photoproduct. (b) Theoretical spectra predicted (at the DFT(B3LYP)/6-311++G(d,p) level) for the cbI (open circles) and cbII (full circles) conformations of methylcarboxy-cyclobutadiene (see Scheme 2). The calculated frequencies were scaled by a factor of 0.978.

\section{Conclusion}

The photochemistry of the matrix-isolated methyl coumalate (a molecule belonging to the hitherto scarcely studied family of 5 -substituted $\alpha$-pyrones) has been investigated in the present study. The structures of the initial compound as well as of the photoproducts were probed by the infrared spectroscopy, supported by the high-level quantum chemical calculations. The exposure of matrix-isolated methyl coumalate to the UV $(\lambda>295 \mathrm{~nm})$ light has been shown to induce two photoisomerisation reactions. The isomerisation to the Dewar form of the compound turned out to dominate, whereas the alternative reaction was the cleavage of the $\alpha$-bond $(\mathrm{C}-\mathrm{O})$ generating the open-ring aldehyde-ketene. Both processes were determined to be reversible, the position of the photoequilibrium being dependent on the excitation light. Upon $\lambda>200 \mathrm{~nm}$ irradiation, the monomeric Dewar species was found to undergo decarboxylation, with a production of the methoxycarbonyl substituted cyclobutadiene. Both the vibrational characterisation of the detected species and the observation of their infrared spectra were carried out for the first time.

\section{Acknowledgement}

This work was financially supported by 'Fundação para a Ciência e a Tecnologia' (project POCTI/QUI/59019/2004 and Grant SFRH/BPD/1661/2000).

\section{References}

[1] V.J. Ram, P. Srivastava, Curr. Org. Chem. 5 (2001) 571.

[2] G.P. McGlacken, I.J.S. Fairlamb, Natural Product Rep. 22 (2005) 369.

[3] I.J.S. Fairlamb, L.R. Marrison, J.M. Dickinson, F.J. Lu, J.P. Schmidt, Bioorg. Med. Chem. 12 (2004) 4285.
[4] L.R. Marrison, J.M. Dickinson, I.J.S. Fairlamb, Bioorg. Med. Chem. Lett. 13 (2003) 2667.

[5] D.A. Brown, J. Photochem. Photobiol. B 63 (2001) 148.

[6] J.M. Fu, S.W. Dusza, A.C. Halpern, J. Am. Acad. Dermatol. 50 (2004) 706

[7] G.D. Bandow, J.Y.M. Koo, Int. J. Dermatol. 43 (2004) 555.

[8] J. Barbagallo, C.T. Spann, W.D. Tutrone, J.M. Weinberg, Cutis 68 (2001) 345 .

[9] P.D. Forbes et al., Front. Biosci. 8 (2003) D848.

[10] W. Ma, M. Wlaschek, C. Hommel, L.A. Schneider, K. ScharffetterKochanek, Exp. Gerontol. 37 (2002) 1197.

[11] H. Honigsmann, Clin. Exp. Dermatol. 26 (2001) 343.

[12] W.H. Pirkle, L.H. McKendry, J. Am. Chem. Soc. 91 (1969) 1179.

[13] O.L. Chapman, C.L. McIntosh, J. Pacansky, J. Am. Chem. Soc. 95 (1973) 244.

[14] R.G.S. Pong, J.S. Shirk, J. Am. Chem. Soc. 95 (1973) 248.

[15] A. Krantz, J. Am. Chem. Soc. 96 (1974) 4992.

[16] B.S. Huang, R.G.S. Pong, J. Laureni, A. Krantz, J. Am. Chem. Soc. 99 (1977) 4154.

[17] S. Breda, I. Reva, L. Lapinski, R. Fausto, Phys. Chem. Chem. Phys. 6 (2004) 929.

[18] C.L. McIntosh, O.L. Chapman, J. Am. Chem. Soc. 95 (1973) 247.

[19] S. Breda, L. Lapinski, I. Reva, R. Fausto, J. Photochem. Photobiol. A 162 (2004) 139.

[20] R.D. Rieke, R.A. Copenhafer, Tetrahedron Lett. (1971) 879.

[21] S. Breda, L. Lapinski, R. Fausto, M.J. Nowak, Phys. Chem. Chem. Phys. 5 (2003) 4527.

[22] V. Kvita, W. Fischer, Chimia 46 (1992) 457.

[23] V. Kvita, W. Fischer, Chimia 47 (1993) 3.

[24] H. Javaheripour, D.C. Neckers, J. Org. Chem. 42 (1977) 1844.

[25] I.D. Reva, S.G. Stepanian, L. Adamowicz, R. Fausto, Chem. Phys. Lett. 374 (2003) 631.

[26] H. Rostkowska, L. Lapinski, A. Khvorostov, M.J. Nowak, J. Phys. Chem. A 109 (2005) 2160.

[27] A.D. Becke, J. Chem. Phys. 98 (1993) 5648.

[28] C.T. Lee, W.T. Yang, R.G. Parr, Phys. Rev. B 37 (1988) 785.

[29] S.H. Vosko, L. Wilk, M. Nusair, Canad. J. Phys. 58 (1980) 1200.

[30] M.J. Frisch et al., Gaussian 98, Revision A.9, Gaussian, Inc., Pittsburgh, PA, 1998.

[31] R.L. Redington, J. Chem. Phys. 109 (1998) 10781. 